\title{
Low Cost Osseointegrated Implant System for Developing Countries
}

\author{
Kayla M. Foyt, Jason Roberts \\ Division of Otolaryngology/Head and Neck Surgery, Albany Medical Center, Albany, NY, USA \\ Email: jayrob0723@gmail.com
}

Received May 29, 2013; revised June 22, 2013; accepted July 3, 2013

Copyright (C) 2013 Kayla M. Foyt, Jason Roberts. This is an open access article distributed under the Creative Commons Attribution License, which permits unrestricted use, distribution, and reproduction in any medium, provided the original work is properly cited.

\begin{abstract}
Objective: Osseointegrated hearing implants have been readily available and extensively used in developed countries for over twenty years. Despite a great need for this technology, use of these implants in developing countries has been limited due to the prohibitive cost of the surgical implant system as well as the implant devices themselves. To address this problem we have developed a drill and implant system that mimics the currently available instrumentation using materials that are readily available at a fraction of the cost. Study Design: Proof of Concept. Methods: The construction of the drill and implant system will be described. Testing of the system so that it adhered to Cochlear's ${ }^{\mathrm{TM}}$ Bone anchored hearing aid system specifications including the drill speed and torque settings were measured. Temperature readings were also recorded during procedures performed on human cadaveric temporal bones. Results: The speed of the drill with the guide drill and countersink drill bits ranged from 1400 revolutions per minute (rpm) to $2300 \mathrm{rpm}$ 's. The temperature change of the room temperature temporal bones varied from 0 degrees to +0.2 degrees Fahrenheit during the drilling process with both drill bits. All five implants were stable in the cadaver bone after being checked for initial stability. Conclusion: This low cost implant system may make the BAHA technology more easily available to developing countries pending future studies with animal models.
\end{abstract}

Keywords: Quality of Life; Implants; Otology; BAHA; Hearing Loss

\section{Introduction}

The Bone Anchored Hearing Aid (BAHA) was first implanted in 1977 by Dr Anders Tjellström in Sweden. The BAHA was further refined and implanted in 14 patients by him in 1981 for the treatment of conductive hearing loss in the setting of chronic otitis media [1]. Since then, over 100,000 implant procedures have been performed worldwide for a variety of hearing disorders. Unfortunately, the vast majority of these implants have been performed mostly in developed countries that can afford the technology. The incidence of conductive hearing loss that can be corrected with osseointegrated implants in the developing world is not known. However, the number is likely much higher than in the United States due to a lack of technology or finances available for advanced otologic surgeries for hearing restoration such as ossicular chain reconstruction. The BAHA is a reliable method of auditory rehabilitation and improves the quality of life for

\footnotetext{
*All work was performed at Albany Medical College Anatomy Lab; No financial support was necessary for this project.

\#Corresponding author.
}

patients with middle and external ear deformities [2-7].

Further modifications of this Cochlear ${ }^{\mathrm{TM}}$ osseointegrated hearing device continue to develop and Oticon ${ }^{\mathrm{TM}}$ corporation has developed a device on the same principle. Current BAHA implantation can cost as much as $\$ 30,000$ in western countries. The cost of the implant drill system alone is $\$ 10,000$ (BAHA order form, Cochlear ${ }^{\mathrm{TM}}$ Corporation). The current BAHA implant system that is available from Cochlear ${ }^{\mathrm{TM}}$ Corporation is a combination of a precision single speed drill and a variable selective torque wrench. The drill is set between two modes: drill and torque wrench. The drill rotates at $2000 \mathrm{rpm}$ to create both the initial guide hole perpendicular to the bone and the subsequent countersink hole at a depth of 3 to 4 $\mathrm{mm}$. This will accommodate the titanium implant (abutment) in the next step. The torque wrench mode utilized to implant the titanium abutment can be set between 0 and $50 \mathrm{Ncm}^{2}$ with typical settings of $40 \mathrm{Ncm}^{2}$ for adult implants and $30 \mathrm{Ncms}$ for pediatric implantations. The drill can be dismantled and autoclaved for sterility (Cochlear Baha3 Surgery Guide, Cochlear ${ }^{\mathrm{TM}} \mathrm{Baha}^{\mathrm{B}}$.) Critical variables in the procedure include not touching the 
abutment threads, irrigation for avoidance of heat production during the drilling process, and a torque setting to ensure that the implant does not strip its thread in the bone. This process is followed systematically to optimize osseointegration.

The success of BAHA implants and their ability to provide optimal bone conducted hearing results from an active bond between the titanium implant and the living bone tissue - a process called osseointegration. In previous studies in oral and orthopedic surgery, the most important factor for osseointegration is the avoidance of heat production by the drill bits in the surrounding bone. For failures occurring within 3 months of insertion, these studies found that bone overheating during the surgery lead to failure of osseointegration. Bone necrosis was found to occur when the bone was heated to a temperature of 47 degrees celcius (116.6 degrees F) for one minute [8]. In order to decrease heat production in the bone, in addition to irrigation, most studies have employed using a minimal load and low speed. However, one study demonstrated that through increasing the load of the drill while increasing the drill speed, they found this created more efficient cutting and less frictional heat [8,9]. Tellstrom adhered to these biological principles of osseointegration for the successful development of the bone anchored hearing aid system [1]. Our drill system was developed with these same principles in mind.

As the indications for these implants continue to broaden and develop in both adults and children, several generations of the BAHA sound system processor have been developed by Cochlear ${ }^{\mathrm{TM}}$ to provide sufficient amplification in different hearing situations [10-12]. We propose a drill and torque wrench system that can be easily constructed from off-the-shelf components at a fraction of the cost of the currently available drill system. Through using explanted Cochlear ${ }^{\mathrm{TM}}$ BAHAs, performance measurements were obtained including: drill speed; temperature variations in surrounding bone; ease of use of the instruments; and implant stability. The drill bits could be autoclaved for sterility and a sterile shield for the drill and torque wrench was devised.

\section{Materials and Methods}

Determination was made through the Office of Research Affairs at Albany Medical Center that this project does not involve human subject research and so does not require approval by the IRB.

Various drill systems that would be appropriate for this application were investigated. We investigated several combined drill and torque wrench systems that could mimic the performance of the standard osseointegrated drill system. No drill and torque wrench system was available off the shelf that was low cost, lightweight, and precise enough for the BAHA procedure to be described.
The equipment was therefore purchased as two separate components: a drill and a manual variable torque wrench. We used previously used (one time) sterilized Cochlear ${ }^{\mathrm{TM}}$ drill bits as well as explanted Cochlear ${ }^{\mathrm{TM}}$ BAHA abutments.

The experimental procedure was performed on human cadaveric temporal bones. Drilling was performed with aggressive saline irrigation similar to the recognized routine surgical procedure. The temporal bones were at room temperature prior to doing any of the drilling for the speed and heat measurements. Drills were compared and evaluated for precision, compact design, drill speed, and compatibility with the existing Cochlear ${ }^{\mathrm{TM}}$ BAHA drill bits. We selected a lithium battery powered Dremel $^{\mathrm{TM}}$ drill with variable speed control (Dremel Stylus ${ }^{\mathrm{TM}}$, Dremel, USA). This tool has worldwide distribution and availability (www.dremel.com) In addition, it is an inexpensive ( $\sim$ \$5.00 USD), lightweight and precise drill that can be easily wrapped in a sterile cover. It has a low torque motor that spins at $5000 \mathrm{rpm}$ at its lowest setting. While the drill rotates a higher speed than the Cochlear ${ }^{\mathrm{TM}}$ BAHA drill, we felt that the low torque property of the drill in bone would slow the drill bit to acceptable levels. The drill fits the Cochlear ${ }^{\mathrm{TM}}$ BAHA drill bits without modification. Rotation speed during bone drilling with the guide drill and countersink was measured by a strobe (Cen-Tech Digital Photo Tachometer DT-838). The heat production in the surrounding bone during bone drilling was measured by a precision surface temperature probe (Extech Dual input Digital Thermometer Model 421502). The implant procedure was otherwise not modified from the traditional surgery as described previously by Cochlear ${ }^{\mathrm{TM}}$.

Several electric torque wrenches were investigated for this study. No electric tool that satisfied the requirements of low cost, precision, desired torque range, and portability could be found. Therefore, a hand driven precision adjustable torque wrench was chosen (Screwdriver 61 NSM, CDI Torque Products) that was ideal for the surgical procedure.

\section{Results}

Measurements were performed on human cadaveric temporal bones at room temperature utilizing the drill system as described in the materials and methods section (Figure 1). The Dremel drill was on a setting of \#1 throughout the testing. The guide drill revolutions per minute (rpm) were measured initially with and without contact on the temporal bone (Figure 2). The rpm's ranged between 4800 to 5200 without contact and $2000 \mathrm{rpm}$ with initial skull contact. Five tests with skull contact were performed with rpms ranging from 1400 to $2000 \mathrm{rpms}$ with no test measuring over $2000 \mathrm{rpms}$ at a setting of 1 on the drill (Table 1). To assure we consistently meas- 
Table 1. Measurement of revolutions per minute (rpm) with the guide drill bit on temporal bone.

\begin{tabular}{cc}
\hline Test & Rotations per minute \\
\hline 1 & 1400 \\
2 & 1800 \\
3 & 2000 \\
4 & 1800 \\
5 & 1800
\end{tabular}

Drill speed without temporal bone contact: $5200 \mathrm{rpm}$; Drill speed with initial temporal bone contact: $2000 \mathrm{rpm}$.

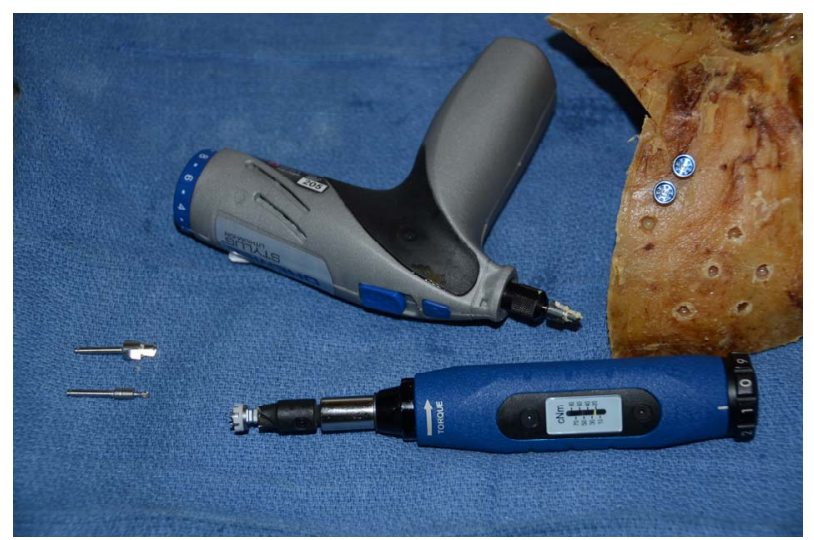

Figure 1. Drill and torque wrench system with associated drill bits, torque bit, and abutments in a temporal bone.

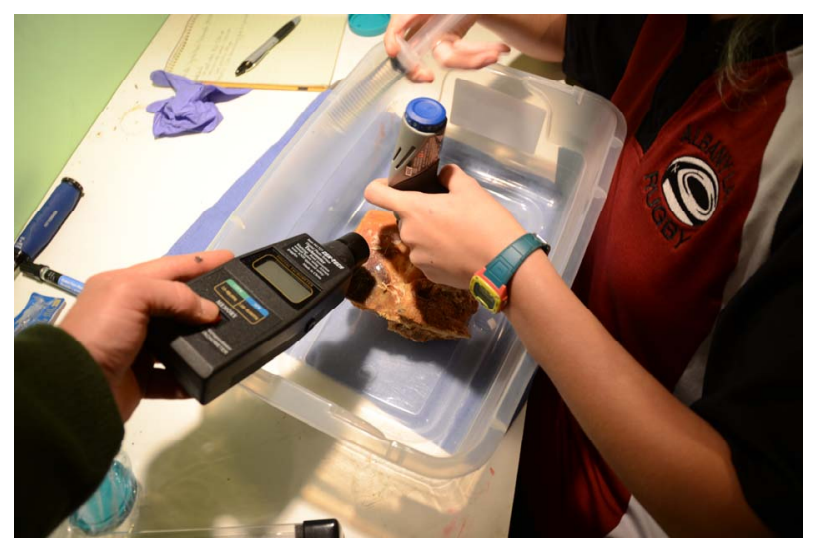

Figure 2. Utilizing the Cen-Tech digital photo tachometer DT-838 to measure the revolutions per minute during drilling of the temporal bone utilizing the guide drill bit and countersink drill bit.

ured the rpms, we utilized the weight of the drill with minimal pressure because we noted that enough pressure could be applied to stop the drill completely. We allowed the drill speed and drill bit to perform the work in order to drill the guide hole precisely. The lowest rpm was noted initially on test 1 when too much pressure was applied with the guide drill.

After the guide drill hole was performed, the second step of the BAHA drill system involved the countersink portion of the procedure. Five tests were performed with the countersink and the rpm's were measured. We found the rpms in each of the five tests to range from 1600 to 2300 while drilling in the cadaveric temporal bones (Table 2).

The third portion of the test involved temperature measurements of the temporal bone utilizing the digital thermometer. Prior to applying the temperature probe to the temporal bone (Figure 3), variations in the digital thermometer were measured with irrigation. Temperature measurements varied from no change to -0.2 degrees with irrigation to +4 degrees without irrigation (Table 3 ). When the temperature probe was applied to the bone, the bone temperature measured $71.5 \mathrm{deg} F$ which was consistent with the room temperature of the testing center. As stated previously, the drill was set at \#1 setting and 5 measurements were run with the guide drill and countersink drill to evaluate temperature changes. With continuous irrigation with sterile water, the increase in temperature ranged from 0 degrees to 0.2 degrees (Table 4).

The final portion of the experiment involved using the

Table 2. Measurement of revolutions per minute with countersink drill bit on temporal bone.

\begin{tabular}{cc}
\hline Test & Rotations per minute \\
\hline 1 & 1800 \\
2 & 2000 \\
3 & 1900 \\
4 & 1600 \\
5 & 2300 \\
\hline
\end{tabular}

Table 3. Temperature probe measurements sensitivity under irrigation (not attached to temporal bone).

\begin{tabular}{cc}
\hline Test & Initial temperature $\left(72.3^{\circ} \mathrm{F}\right)$ \\
\hline 1 & No change \\
2 & -0.2 degrees \\
3 & No change \\
4 & -0.1 degrees \\
5 & +4 degrees ( no irrigation) \\
\hline
\end{tabular}

Table 4. Temperature probe measurements with probe attached to temporal bone.

\begin{tabular}{ccc}
\hline Test & $\begin{array}{c}\text { Guide Drill } \\
\text { Initial Temperature }\left(65.5^{\circ} \mathrm{F}\right)\end{array}$ & $\begin{array}{c}\text { Countersink Drill } \\
\text { Initial Temperature }\left(71.5^{\circ} \mathrm{F}\right)\end{array}$ \\
\hline 1 & +0.1 degrees & +0.2 degrees \\
2 & No change & +0.1 degrees \\
3 & +0.1 degrees & +0.1 degrees \\
4 & +0.2 degrees & No change \\
5 & No change & No change \\
\hline
\end{tabular}




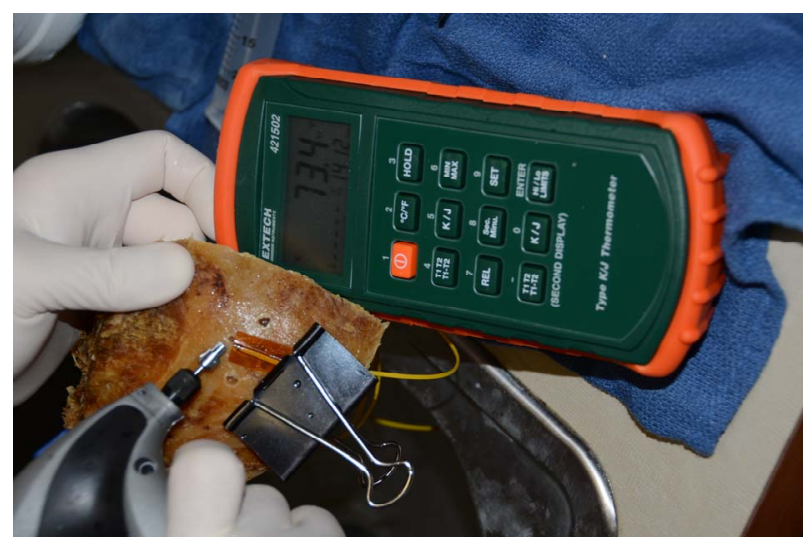

Figure 3. Utilizing the Extech dual input digital thermometer model 421502 to measure the temperature of the temporal bone adjacent to the drill hole while drilling with the guide drill and countersink drill.

torque wrench to determine what setting to determine if the implant could successfully be drilled and was grossly stable. The abutment could be easily adapted to this torque wrench for placement. The setting of the torque wrench was approximately $25 \mathrm{Ncm}$ which is the setting recommended by the Cochlear Surgery Guide (Cochlear ${ }^{\mathrm{TM}} \mathrm{Baha}^{\mathbb{B}}$ ) for compromised or soft bone. In all 5 temporal bones, the torque wrench was able to drill the Cochlear ${ }^{\mathrm{TM}}$ BAHA abutments. The implanted abutments were grossly stable with manual manipulation (Figure 4).

\section{Discussion}

The World Health Organization reports that over 275 million people worldwide suffer from some degree of hearing loss with a majority of those people $(80 \%)$ in low to middle income nations (WHO fact sheet). Hearing loss can have an enormous impact on the quality of life as well as impact on the productivity of an individual. Developed countries are able to improve hearing through the various technologies available ranging from cochlear and brainstem implants to ossicular chain reconstructions. Bone anchored hearing aids have also been available in these developed countries for over 20 years as a reliable method of auditory rehabilitation that improves the quality of life for patients with conductive, sensorineural or mixed hearing loss due to many causes [2-7]. Since its creation, several generations of the BAHA sound system processors have been developed by Cochlear to provide sufficient amplification in different hearing situations [10-12]. This technology, which relies on the unique concept of osseointegration, requires expensive and standardized equipment for its use and therefore, developing countries are unable to utilize this technology where it would provide significant benefit. This technology could be more beneficial in those developing countries as it

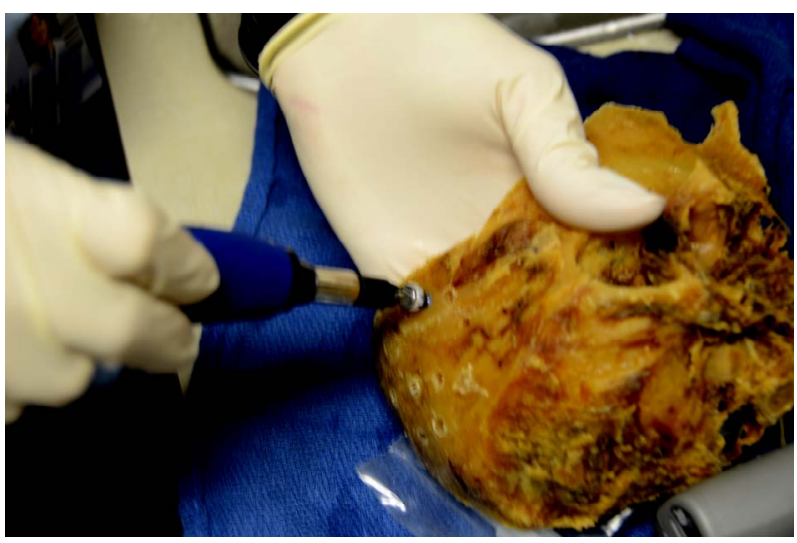

Figure 4. Utilizing the manual torque wrench to the implant the BAHA abutments.

would bypass the middle ear in patients' with a wide array of pathologies. Unfortunately, the cost of the technology to implant these BAHAs is prohibitive. Our goal was to develop a system that could be utilized in a sterile manner in these developing countries so that this valuable hearing technology could be expanded.

Through our temporal bone studies with our off-theshelf surgical instruments, we have shown that this is a possibility. With temporal bone contact, the speed of the Dremel drill proved to be comparable to the standard Cochlear ${ }^{\mathrm{TM}}$ BAHA instrumentation through the utilization of sterilized previously used Cochlear ${ }^{\mathrm{TM}}$ sharp drill bits. At a setting of \#1 on the the drill, the highest speed recorded was $5200 \mathrm{rpms}$ without bone contact, yet with initial skull contact the drill speed dropped to $2000 \mathrm{rpms}$. We attempted to utilize \#2 setting on the drill but this tended to strip the bone during drilling. Therefore, we utilized a setting of \#1 on the drill throughout the study for our measurements. The lowest speed recorded was found to be $1400 \mathrm{rpms}$ which was due to applying too much pressure. We believe that the \#1 drill speed is appropriate for the guide drill and countersink drill. Other rotary drills systems could be tested for application of this Cochlear technology. However, the Dremel drill consistently provided us with an adequate rotation speed for the guide drill and countersink drill. In addition, through utilizing the standardized digitial photo tachometer, the rpm's of the drill did not vary considerably.

For the heat measurements, we utilized a standardized digital temperature probe on the room temperature temporal bones. Prior to attaching the probe to the temporal bone, the variability of the thermometer was minimal with constant irrigation. The thermometer was secured adjacent to the hole that was being drilled in order to accurately measure any change in the temperature of the temporal bone. Although this would not record the temperature of the bone in the drill hole, we can assume that this bone temperature was very close to that of the adja- 
cent drill hole. Five runs were performed for measuring the speed and temperature during the guide and countersink drilling. Due to minimal changes in the values during these five runs, no further runs were deemed necessary for this proof of concept design study. We did use human temporal bones and proved that this concept is applicable. The compromised and softer nature as well as the lower temperature of the human cadaveric temporal bones make it necessary to carry out these same studies in animal models prior to performing this in humans.

The abutments were placed with a manual torque wrench at the settings specified by Cochlear ${ }^{\mathrm{TM}}$. No heat measurements were performed during this portion of the procedure but the speed was minimal during this part of the study and would not impede osseointegration. Although osseointegration was not determined with this drill system, the abutments were secure with manual manipulation. Adhering to the implant surgical guidelines and minimizing heat production during the drilling process, osseointegration should not be a problem utilizing this drill system.

A factor that could impede osseointegration is previously utilized explants that had been sterilized. The Cochlear ${ }^{\mathrm{TM}}$ guidelines are to never touch the threaded portion of the titanium abutment due to its titanium oxide coat and the reported important factor this plays in osseointegration. Studies have shown that the oxidized coating is an important factor to increase the surface area which assists with osseointegration [13]. To the best of our knowledge, no studies were found in the literature which determine the extent of osseointegration after sterilizing these explanted models. Our belief is that osseointegration will occur without the titanium oxide coating on the implants.

We developed a simple method of sterilizing these tools. The drill bits utilized with the guide Dremel drill could be sterilized in the autoclave which is available in most developing countries. The Dremel drill was small and although this could not be placed in the autoclave, different techniques to keep this drill sterile were attempted. We attempted to use the plastic covering from the intra-operative ultrasound and intraoperative lymphoscintigrophy scan used for parathyroid localization. These sterile drapes are prohibitive however in that they are specific to these instruments and not readily available in developing countries. Therefore, we were able to use a sterile glove which is more readily available. We found that placing the Dremel into the sterile glove trough one of the finger tips, size 7 or higher, and puncturing a small hole in the tip with the drill allowed us to keep the drill sterile. The glove could be changed for subsequent use of the drill. The actual sterility and bacterial counts after subsequent use of the drill is something that should be measured prior to expanding this methodology to hu- mans.

The cost of the drill was $\$ 75.00$ and Dremel is an international company with distribution centers worldwide. This drill could easily be obtained for developing countries. The cost of the drill bits and abutments could be prohibitive if they were to be purchased from Cochlear ${ }^{\mathrm{TM}}$. Fortunately, these tools could be donated and sterilized as they were done for this study. In terms of the abutments, the previous patients' abutments were removed for various reasons and used in this study. Most companies and otologists have a handful of explanted abutments or prior generations of abutments that could be made available to developing countries. In addition, the drill bits used in our study were only used once previously in the operating room setting. These bits are extremely sharp after only one use and could be sterilized for use in future implants. In our study, we used the same drill bit and countersink for 5 runs each and it did not alter the temperature or rpms. These additional tools after being sterilized could be donated or collected from previous surgeries to be taken or sent to developing countries. It is important to emphasize that sterility of previously utilized drill bits, and abutments including bacterial counts should be the subject of future animal studies.

\section{Conclusion}

We developed this novel system using off-the-shelf products and measured different aspects of this system to assure it complied with the specifications used by the Cochlear ${ }^{\mathrm{TM}}$ BAHA. We also demonstrated this systems' applicability and ease of use. However, prior to sharing this BAHA technology with developing countries worldwide, future animal studies are necessary for utilizing our system.

\section{REFERENCES}

[1] A. Tjellstrom, et al., "Osseointegrated Titanium Implants in the Temporal Bone," American Journal of Otolaryngology, Vol. 2, No. 4, 1981, pp. 304-310.

[2] S. Bouhabel, P. Arcand and I. Saliba, "Congenital Aural Atresia: Bone-Anchored Hearing Aid vs. External Auditory Canal Reconstruction," International Journal of Pediatric Otorhinolaryngology, Vol. 76, No. 2, 2012, pp. 272-277. doi:10.1016/j.ijporl.2011.11.020

[3] Y. Fan, et al., "Efficancy in Patient with Outer and Middle Ear Deformities of Using Bone-Anchored Hearing Aids," Chinese Journal of Otorhinolaryngology Head and Neck Surgery, Vol. 47, No. 4, 2012, pp. 265-269.

[4] A. D. Snik, et al., "The Bone-Anchored Hearing Aid for Children: Recent Developments," International Journal of Audiology, Vol. 47, No. 9, 2008, pp. 554-559. doi: $10.1080 / 14992020802307354$

[5] C. M. Steward, J. H. Clark and J. K. Niparko, "BoneAnchored Devices in Single-Sided Deafness," Advances 
in Oto-Rhino-Laryngology, Vol. 71, 2011, pp. 92-102. doi:10.1159/000323589

[6] M. K. S. Hol, et al., "Bone-Anchored Hearing Aids in Unilateral Inner Ear Deafness: An Evaluation of Audiometric and Patient Outcome Measurements," Otology \& Neurotology, Vol. 26, No. 5, 2005, pp. 999-1006. doi:10.1097/01.mao.0000185065.04834.95

[7] R. M. Janssen, P. Hong and N. K. Chadha, "Bilateral Bone-Anchored Hearing Aids for Bilateral Permanent Conductive Hearing Loss: A Systematic Review," Otolaryngology—Head and Neck Surgery, Vol. 147, No. 3, 2012, pp. 412-422.

[8] S. A. Hadi, N. Ashfaq, A. Bey and S. Khan, "Biological Factors Responsible for Failure of Osseointegration in Oral Implants," Biology and Medicine, Vol. 3, No. 2, 2011, pp. 164-170.

[9] D. L. Brisman, "The Effect of Speed, Pressure, and Time on Bone Temperature during the Drilling of Implant
Sites," International Journal of Oral \& Maxillofacial Implants, Vol. 11, No. 1, 1996, pp. 35-37.

[10] A. F. Snik, et al., "Consensus Statements on the BAHA System: Where Do We Stand at Present," Annals of Otology, Rhinology, and Laryngology, Vol. 114, No. 12, 2005, pp. 2-12.

[11] S. Roman, R. Nicollas and J. M. Triglia, "Practice Guidelines for Bone-Anchored Hearing Aids in Children," European Annals of Otorhinolaryngology, Head and Neck Diseases, Vol. 128, No. 5, 2011, pp. 253-258.

[12] C. A. J. Dun, et al., "An Overview of Different Systems: The Bone-Anchored Hearing Aid," Advances in OtoRhino-Laryngology, Vol. 71, 2011, pp. 22-31.

[13] R. Branemark, et al., "Osseointegration in Skeletal Reconstruction and Rehabilitation: A Review," Journal of Rehabilitation Research \& Development, Vol. 38, No. 2, 2001, pp. 175-181. 